\title{
Raspberry Pi Based Battle Field Robot Using Dijkstra's Algorithm
}

\author{
S. Lokesh, Akash G, Gangadharan S, Prabhu A C, Vivek kumar sharma P
}

\begin{abstract}
The robot is intended to follow protests by turning left and ideal to keep the article in sight and driving forward and in reverse to keep up a consistent separation between the robot and the item. Pictures are obtained through the camera of a Raspberry-pi gadget which is appended to the robot. The camera is appended to servos on the robot which enable the camera to skillet and tilt. A few picture handling methods are utilized to distinguish the area of the article being followed in the pictures. The vehicle route utilizing the information transmission time is expanded with the convention standard. Correspondences between two hubs (equipment and application) are practiced through IEEE 802.15.4. The client can give the source and goal hub address to the server segment. Utilizing $Q R$ code by executing DIJSKTRA calculation the most brief way can be effectively decided. Metal discovery should be possible by utilizing inductive closeness sensor. Ultrasonic sensor is utilized for finding the separation between the robot and article to be picked.
\end{abstract}

Keywords: Dijkstra algorithm, Raspberry-pi device, Analogue-to-digital converter (ADC)

\section{INTRODUCTION}

Science has conveyed out pondering advancements to ease human life. Mechanical autonomy is one of its parts, which has made human life simpler and decreased the outstanding burden. It has additionally empowered us to diminish the support of human in unsafe works. These days robots are being utilized for different reason in businesses, labs, Space and furthermore in front line. Individuals are sending robots to places where man can barely go like in space, submerged, bomb encompassed territories. Remote correspondence framework has turned out to be one of the fundamental highlights for business items and a prevalent research theme inside the last ten years[1-6]. There are presently more cell phone memberships than wired-line memberships. Recently, one region of business intrigue has been minimal effort, low power, and short separation remote correspondence utilized for individual remote systems. Innovation progressions are giving littler and more practical gadgets for incorporating computational handling, remote correspondence, and a large group of different functionalities. In single-robot restriction, the area of the robot is deduced dependent on its movement controls and estimations given a shade of the scene. The current agreeable restriction calculations for the most part accept that the world is static[7-13].

Revised Manuscript Received on July 22, 2019.

S. Lokesh, Akash, Assistant Professor, Department of Electronics and Communication Engineering, VelTech, Chennai-600062

G, Gangadharan, Assistant Professor, Department of Electronics and Communication Engineering, VelTech, Chennai-600062

S, Prabhu A C, Assistant Professor, Department of Electronics and Communication Engineering, VelTech, Chennai-600062

Vivek kumar sharma P, Assistant Professor, Department of Electronics and Communication Engineering, VelTech, Chennai-600062

\section{PROPOSED SYSTEM}

In this work, we have proposed the robot that is intended to follow protests by turning left and ideal to keep the item in sight and driving forward and in reverse to keep up a consistent separation between the robot and the article. Pictures are gained through the camera of a Raspberry-pi gadget which is appended to the robot. The camera is joined to servos on the robot which enable the camera to skillet and tilt. A few picture preparing systems are utilized to distinguish the area of the item being followed in the pictures. The vehicle route utilizing the information transmission time is expanded with the convention standard. Correspondences between two hubs (equipment and application are practiced through IEEE 802.15.4. The client can give the source and goal hub address to the server area. Utilizing QR code by executing Dijkstra calculation the briefest way can be effectively decided. Metal location should be possible by utilizing inductive closeness sensor.

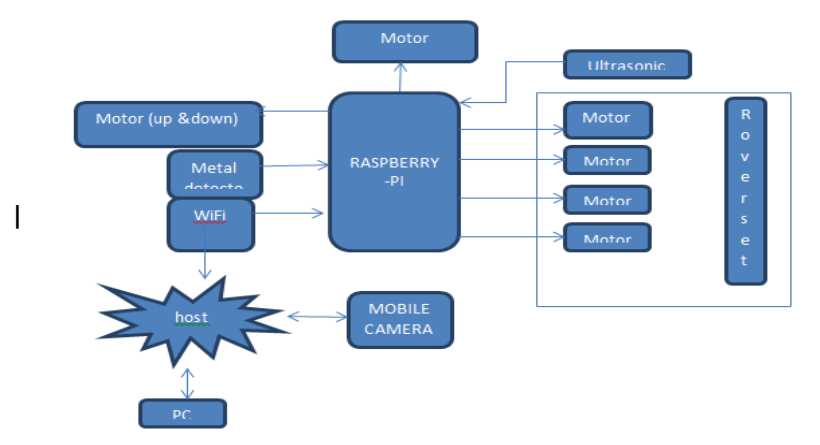

Fig 1: block diagram of raspberry pi based battle field robot In the above square graph (Fig 1) the versatile camera recognized the Color code and travel to the article to be picked utilizing ultrasonic sensor, it is utilized to know the separation between the robot and the item .The pc investigation the article identification and send the hinder to the Raspberry-pi. In light of the interfere with the engine move and the picks just when the metal indicator identifies the metal article and spot it to the goal area. The module comprise of three D.C engines to be specific one arm engine and two wheel engine, one ultrasonic sensor to follow the separation between the article and the robot, one inductive vicinity sensor to recognize the metal on the item to be picked, versatile camera is utilized for item shading following by utilizing IP webcam application .IP address of the webcam to be opened in internet searcher, camera identifies the shade of the item and moves towards it and metal identifying

sensor

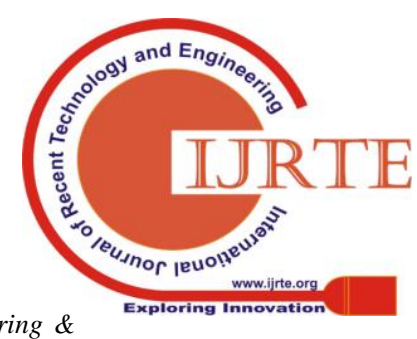




\section{Raspberry Pi Based Battle Field Robot Using Dijkstra's Algorithm}

distinguishes the metal and picks it. QR code is utilized for acknowledgment and djiktra calculation is which finds a most brief way accessible to move towards its goal.

Raspberry Pi3

In this work we are utilizing Latest adaptation of Raspberry for example Raspberry Pi 3The processor at the core of the Raspberry Pi 3 is a Broadcom BCM2837, and the later models of the Raspberry Pi 2. The basic engineering of the BCM2837 is indistinguishable to the BCM2836. The Only noteworthy contrast is the substitution of the ARMv7 quad center group with quad-center ARM Cortex A53 (ARMv8) bunch. The ARM centers keep running at $1.2 \mathrm{GHz}$, making the gadget about half quicker than the Raspberry Pi 2 with an on board memory of $1 \mathrm{~GB}$ RAM. The videocore IV keeps running at $400 \mathrm{MHz}$ appeared in fig 2 .

board and a 16 pi IC base and couple of male header pins. Presently place your IC base on the PCB and imprint that a semi-circle will be the top and numbering should begin notice in Figure. 4. After that place 8 male header pins adjacent to the $\mathrm{CHO}-\mathrm{CH} 7$ pins of the IC base a weld them as show in the underneath Figure 5. Presently take

four male header sticks on the highest point of the IC base and patch them and short VDD and Vref .

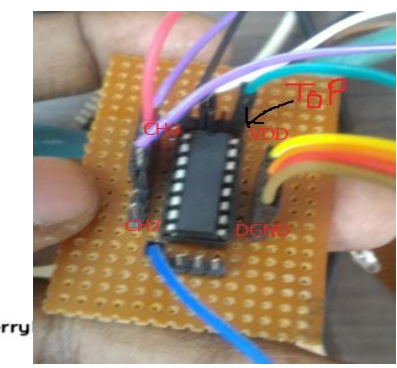

Fig.4 MCP3008 Front View View

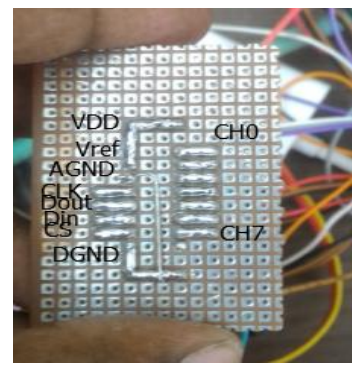

Fig 5: MCP3008 Back

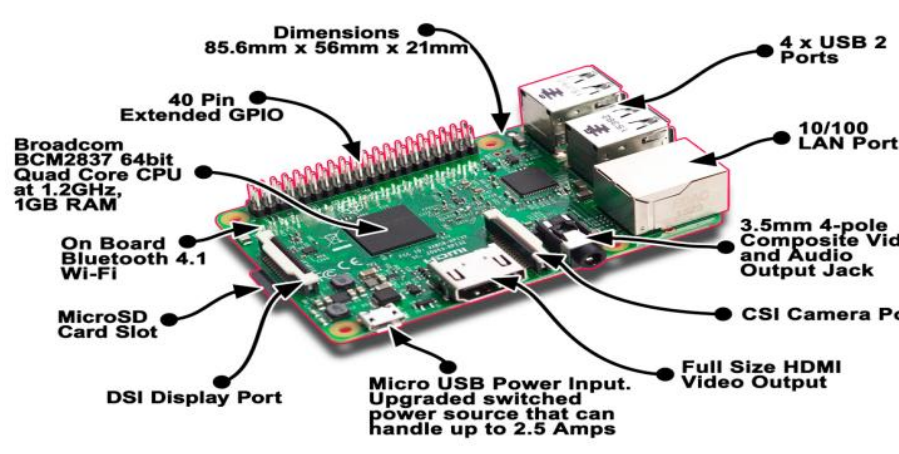

Fig 2: Raspberry Pi3

\section{MICROCHIP ADC}

The MCP3008 is a 10bit 8-channel Analog-to-computerized converter (ADC). It is shabby, simple to interface and doesn't require any extra segments. It utilizes the SPI transport convention which is upheld by the Pi's GPIO header.The clarification of how to utilize a MCP3008 gadget to give 8 simple sources of info which you can use with a scope of sensors is portrayed. The circuit beneath demonstrates the MCP3008 to peruse a temperature and light sensor appeared in fig 3

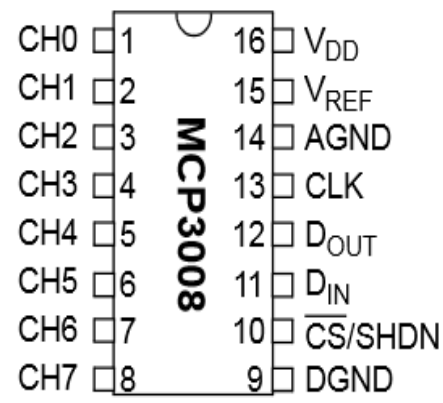

Fig 3: MCP3008 Pin Description

Hardware Making

We need to make a MCP3008 IC base so the association ends up simpler. We need some equipment for example PCB

\section{INDUCTIVE PROXIMITY SENSOR}

deo A security framework is created by utilizing closeness sensor which is named as a metal finder. Thus, a metal identifier is utilized in numerous mechanical or gadgets tasks to identify any present metals which are close-by or the presence of shrouded things inside items. Metal identifiers distinguish iron, nickel, copper, metal, aluminum, tin, lead, gold, silver and bronze. Universally useful metal identifiers can discover covered metal items, for example, gems, coins and other metal articles. "Segregation" is a procedure that recognizes distinctive metal targets or combinations appeared in fig 6 .

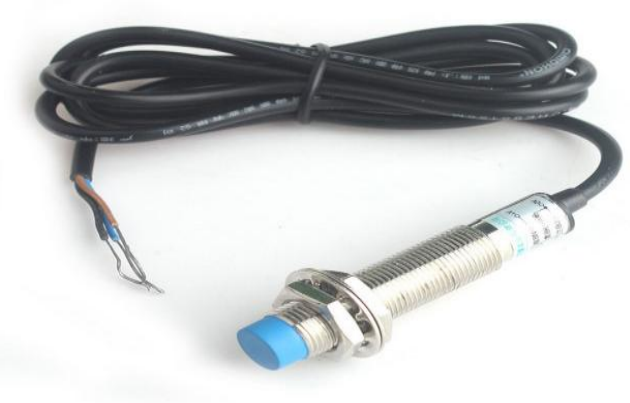

Fig 6 Inductive proximity sensor

\section{ULTRASONIC SENSOR}

Ultrasonic sound vibrates at a frequency above the range of human hearing our ultrasonic sensors, like many others, use a single transducer to send a pulse and to receive the echo. The sensor determines the distance to a target by 
measuring time lapses between the sending and receiving of the ultrasonic pulse shown in fig 7.

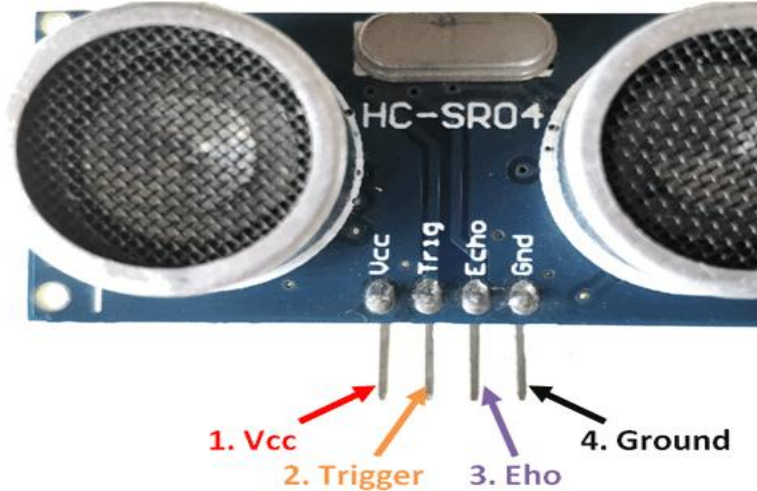

Fig 7: Sensor

\section{DC MOTOR}

A DC engine is any engine inside a class of electrical machines whereby direct flow electrical power is changed over into mechanical power. Regularly, this kind of engine depends on powers that attractive fields produce. Despite the sort, DC engines have some sort of inside system, which is electronic or electromechanical. In the two cases, the bearing of current stream in some portion of the engine is changed occasionally appeared in fig 8.

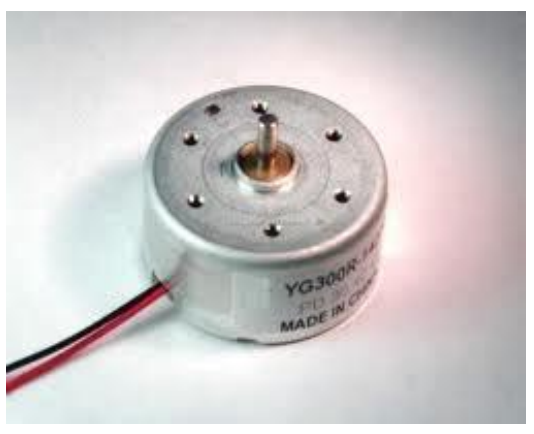

Fig 8: DC Motor

\section{RELAY}

In this work we are going to use to transfers for example one for FAN with $9 \mathrm{~V}$ battery and another for Bulb with $220 \mathrm{~V}$. Beneath we have the hand-off circuit, as in a hand-off we have aggregate of 8 sticks out of which we are utilizing just 5 pins for example two Coil, Com, NO, NC pins. As depicted beneath the stick no begin from the highest point of it where a red circle is made appeared in fig 9

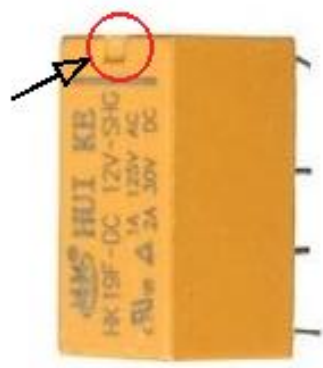

Fig 9: Relay

\section{EXPERIMENTAL RESULTS}

he working model is appeared as follows. Figure 10, lemonstrates the front view. Figure 11, Shows the side view. The robot can be worked by utilizing Raspberry pi is the primary part which controls the development of the robot by controlling the engines. The robot can be worked by utilizing he python program. The transfers go about as an association between the raspberry pi and the engine which controls the wheels of the robot. Transfers control the engine by exchanging the electrical signs from High to low dependent on the directions. The neighborhood have module is associated with raspberry pi which gets guidelines from the Server and passes it on to the raspberry pi.An LCD show Monitor is utilized to show the present guidance that is being kept running in the robot. The robot is controlled a $12 \mathrm{~V} \mathrm{DC}$ engine with $100 \mathrm{rpm}$ the engines are provided with $12 \mathrm{v}$ (7ah) $\mathrm{dc}$ battery. In this the robot is provided with $12 \mathrm{v}$ power supply , a battery is been joined with the meanderer. A neighborhood have server is been arranged with the raspberry pi and it is associated through portable hotspot of same system with same IP address given by the IP webcam application. What's more, Python writing computer programs is coded in raspberry pi board and its been implanted with the robot for continuing the procedure. The portable camera has been set with the wanderer which sends the information of the zone spread and items present in that extend .The article is followed picture detecting and metal location utilizing inductive nearness sensor. Ultrasonic sensor produces the separation count of the item and briefest way for achieving the article is been finished with Dijkstra's calculation .And meanderer starts to move toward the confined territory, object is then picked by the arm in the wanderer connected, from the source and goal way is been instructed ,the wanderer begin to move to the goal way by filtering QR codes set in the dividers. Lastly the article is been put in the goal. At that point the program gets finished and every one of the pieces of information are gotten.

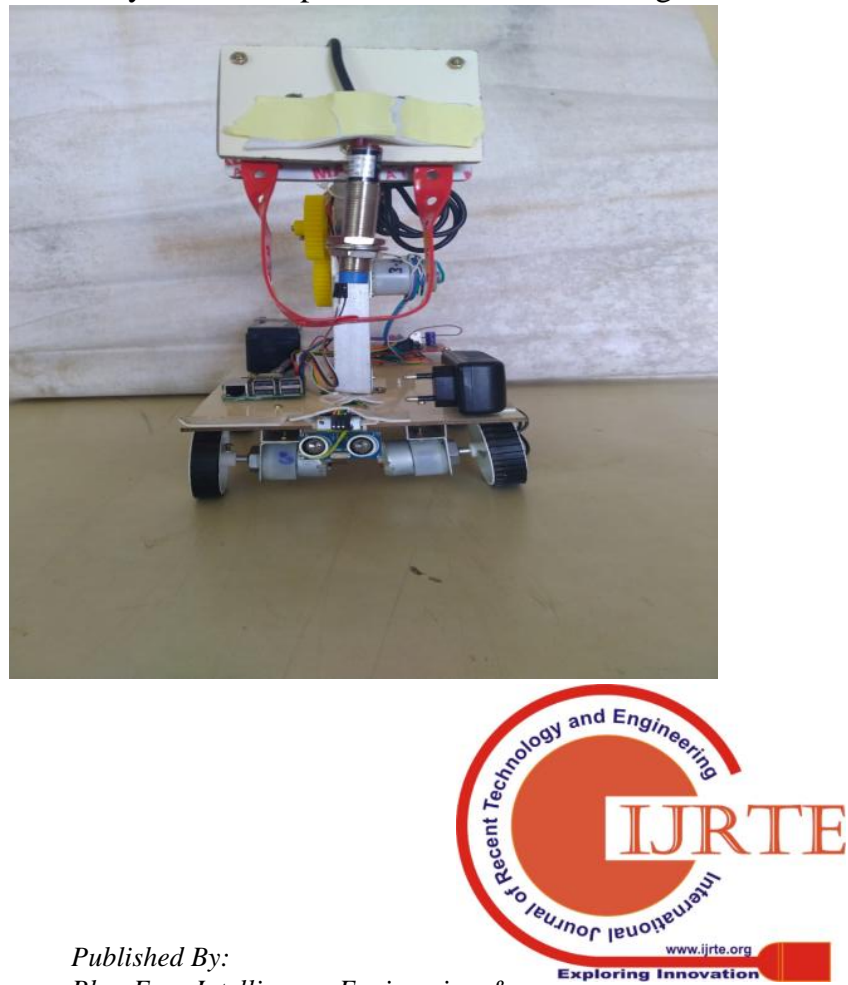

Blue Eyes Intelligence Engineering \& Sciences Publication 


\section{Raspberry Pi Based Battle Field Robot Using Dijkstra's Algorithm}

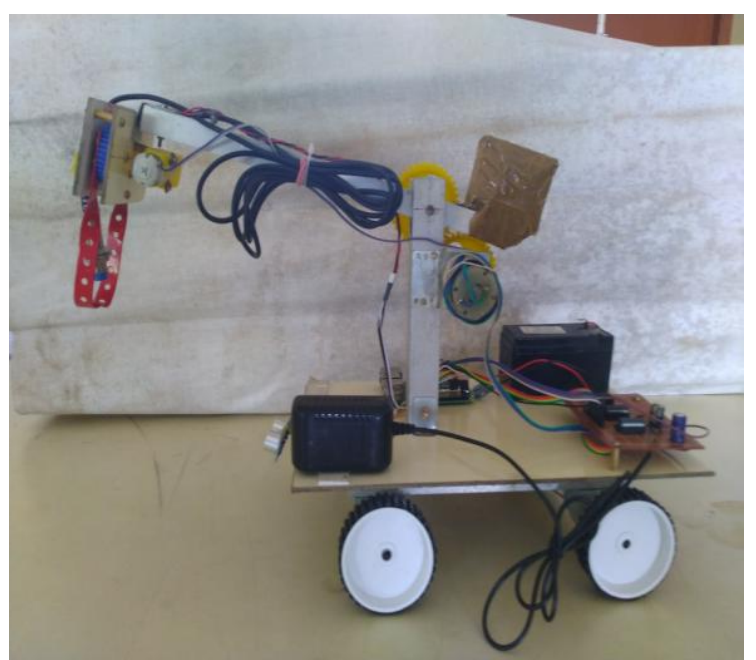

Fig10: A Front View

Fig 11: B Side View

\section{CONCLUSION}

In this work, latest methodology of utilizing Dijkstra's Algorithm for limiting the most brief way in the war zone. A ultrasonic sensor is put for identifying the deterrents in the zone territory. What's more, utilization of metal finder to find the landmines put under the ground, the automated arm is utilized for pick and spot of articles. All the procedure are done through python programming in raspberry pi, the restriction of item and way arranging of the robot is caught by the camera which is associated with the nearby host through wireless association.

\section{REFERENCES}

1. J. Pan, R. Jain, S. Paul, T. Vu, A. Saifullah and M. Sha, "An Internet of Things Framework for Smart Energy in Buildings: Designs, Prototype, and Experiments," IEEE Internet of Things Journal, vol. 2, no. 6, pp. 527-537, 2015.

2. R. Piyare, "Internet of Things: Ubiquitous Home Control and Monitoring System using Android based Smart Phone," International Journal of Internet of Things, vol. 2 no. 1, pp. 5-11, 2013.

3. J. Wan, M.J. O’Grady, G.M.P. O’Hare, "Dynamic Sensor Event Segmentation for Real-Time Activity Recognition in a Smart Home Context," Personal and Ubiquitous Computing, vol. 19, no. 2, pp. 287-301, 2015.

4. K. Afifah, S. Fuada, R.V.W. Putra, T. Adiono, M.Y. Fathany, "Design of Low Power Mobile Application for Smart Home," Proc. of Int. Symposium on Electronics and Smart Devices (ISESD), pp. 127-131, November 2016.

5. Videla and J.J.W. Williams, RabbitMQ in Action Distributed Messaging for Everyone, New York: Manning Publication Co., 2012.

6. Z. R. Lai, D. Q. Dai, C. X. Ren, and K. K. Huang, "Discriminative and compact coding for robust face recognition," IEEE Transactions on Cybernetics, vol. 45, pp. 1900-1912, 2015.

7. Dr. AntoBennet, M, Sankar Babu G, Natarajan S, "Reverse Room Techniques for Irreversible Data Hiding”, Journal of Chemical and Pharmaceutical Sciences 08(03): 469-475, September 2015.

8. Dr. AntoBennet, M , Sankaranarayanan S, Sankar Babu G, “ Performance \& Analysis of Effective Iris Recognition System Using Independent Component Analysis", Journal of Chemical and Pharmaceutical Sciences 08(03): 571-576, August 2015.

9. Dr. AntoBennet, M, Suresh R, Mohamed Sulaiman S, "Performance \&analysis of automated removal of head movement artifacts in EEG using brain computer interface", Journal of Chemical and Pharmaceutical Research 07(08): 291-299, August 2015.

10. Dr. AntoBennet, M "A Novel Effective Refined Histogram For Supervised Texure Classification", International Journal of Computer \& Modern Technology, Issue 01 ,Volume02,pp 67-73, June 2015.
11. Dr. AntoBennet, M, Srinath R,Raisha Banu A,"Development of Deblocking Architectures for block artifact reduction in videos", International Journal of Applied Engineering Research,Volume 10, Number 09 (2015) pp. 6985-6991, April 2015.

12. AntoBennet, M \& JacobRaglend, "Performance Analysis Of Filtering Schedule Using Deblocking Filter For The Reduction Of Block Artifacts From MPEQ Compressed Document Images", Journal of Computer Science, vol. 8, no. 9, pp. 1447-1454, 2012.

13. AntoBennet, M \& JacobRaglend, "Performance Analysis of Block Artifact Reduction Scheme Using Pseudo Random Noise Mask Filtering”, European Journal of Scientific Research, vol. 66 no.1, pp.120-129, 2011.

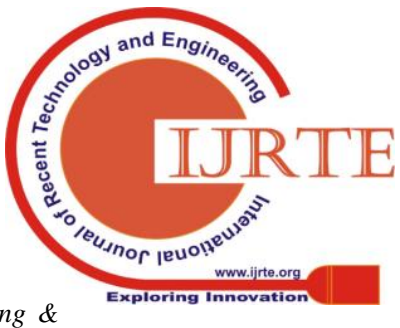

